\title{
Memory, brain and aging: The good, the bad and the promising
}

\author{
by Beth $\mathrm{A}$. Ober
}

\begin{abstract}
A large body of evidence converges on the conclusion that episodic memory (the recollection of personally experienced events) is the only longterm memory system that shows
\end{abstract} significant age-related deficits. Moreover, the brain regions most likely to show age-related volume loss are those most critically involved in episodic memory. Older adult brains may have much greater plasticity (capacity to change) than once believed; for example, neurogenesis (the birth of new neurons), increases in cognitive (including memory) performance, and increases in regional brain volume have all been shown to occur in older adulthood, as a result of physical or mental activity/training. The next wave of research will enhance our understanding of brain plasticity in adulthood and enable specific guidelines for lifestyle or pharmacological treatments that optimize brain and memory functioning well into late adulthood.

W hen middle-aged or elderly adults discuss their health and well-being, one of the most commonly expressed worries is memory problems, including the fear of Alzheimer's disease. This is not surprising, given that memory functions are at the core of our day-to-day intellectual and social activities. Memory enables us to know who we are, as well as what our goals, values and beliefs are. Memory allows us to think about recent and remote events in our lives, and to plan for the future. Moreover, memory enables us to accumulate and make use of vast reservoirs of information about people, objects, places, social customs, language and individual domains of expertise (e.g., birds, wine, astronomy, neuroscience).

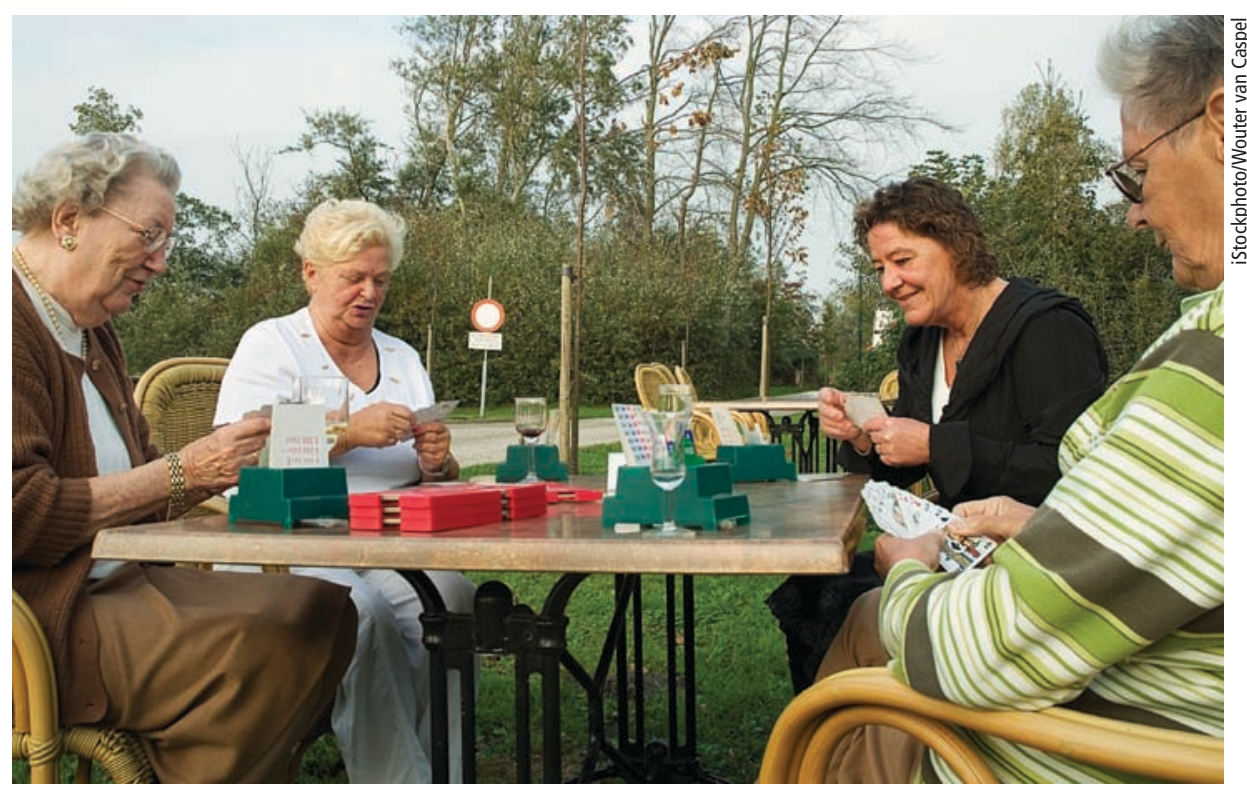

High levels of mental activity, such as playing games like bridge, are predictive of improved cognitive and brain outcomes in older adulthood.

Skills that we have acquired over our lifetimes, most of which can be executed in a fairly automatic manner (e.g., typing, driving, reading, swimming, knitting, playing a musical instrument) also depend on memory.

Adults over 65 years old comprise $11.2 \%$ (4.1 million) of Californians. Due in part to aging baby boomers, California's elderly population is expected to grow twice as fast as the total population to more than 8 million by 2020 (US Census Bureau 2010). Changes in memory function, or even the perception and interpretation of such changes by oneself or by others, can affect day-to-day functioning and wellbeing in a variety of contexts, including family, community and workplace. Each and every one of our actions, interactions, musings, decisions and plans critically depend on memory.

\section{Memory myths vs. reality}

A number of myths persist about memory and aging.

Myth: "Memory abilities will decline in older adulthood to a degree that will significantly affect day-to-day functioning." Rather, about $85 \%$ of adults age 65 and older do not show significant declines in memory ability and are able to live independently.
Myth: "Alzheimer's disease is inevitable in old age." In fact, Alzheimer's disease, which accounts for about $50 \%$ of all dementia cases, occurs in $0.9 \%, 4.2 \%$ and $14.7 \%$, of those 65,75 and 85 years of age, respectively (Brookmeyer et al. 2007). Although the risk of Alzheimer's disease and other dementias increases with age, a minority of older adults are affected.

Myth: "When memory problems occur in normal aging, they involve all aspects of memory." Only one type of long-term memory, known as episodic or event memory, undergoes significant decline in normal aging; other types of long-term memory either remain unaffected or show improvement.

("Normal aging" indicates the absence of dementia or other brain disease, and the ability to live independently.) This misunderstanding reflects the lack of awareness of scientific evidence for several separable types of long-term memory function, each with separable brain underpinnings.

\section{Major types of long-term memory}

Cognitive scientists define short-term memory as that which is available in consciousness (e.g., the sentence that was just spoken, the phone number just dialed), whereas long-term memory 
encompasses any memories that are still available after a brief period ( 30 to 60 seconds) of distraction or disengagement. Information in short-term memory will only be encoded into long-term memory and be available for retrieval after a delay if one is motivated or interested in remembering it. Otherwise, it will quickly be forgotten. When information in short-term memory is manipulated in some way, for example in order to make linkages with information in long-term storage, or in the service of computations, this type of memory is referred to as working memory.

There is general agreement among cognitive psychology and neuroscience scholars on the broad outlines of the behavioral and brain aspects for the following three major types of longterm memory (Schacter and Tulving 1994; Schacter et al. 2000; Squire and Knowlton 2000).

Episodic. Episodic memory involves the conscious recollection of episodes or events, along with a personal context. Recalling a dinner party attended last week, including where the event took place, who was there, what you ate and what the conversations were about, exemplifies episodic memory.

Semantic. Semantic memory involves "world knowledge" in the broadest sense: languages, objects, places, spatial relationships, social norms, facts/trivia, and all sorts of concepts from domains in which one may or may not be an expert. The knowledge brought to the dinner party about the names, backgrounds and interests of the guests is part of semantic memory.

Procedural. Unlike both episodic and semantic memory, procedural memory is not easily accessible to conscious awareness or verbal description and evaluation. Procedural memory includes: (1) sensory-motor skills such as typing, bicycle riding and piano playing; (2) learning and memory for procedures, sequences and rules to solve tasks and puzzles; and (3) repetition priming, an increase in speed and/ or accuracy when identifying a word or picture on repeated presentation as compared to its initial presentation, even when the item is degraded and the presentations are separated by many days (fig. 1). Driving skills used to get to
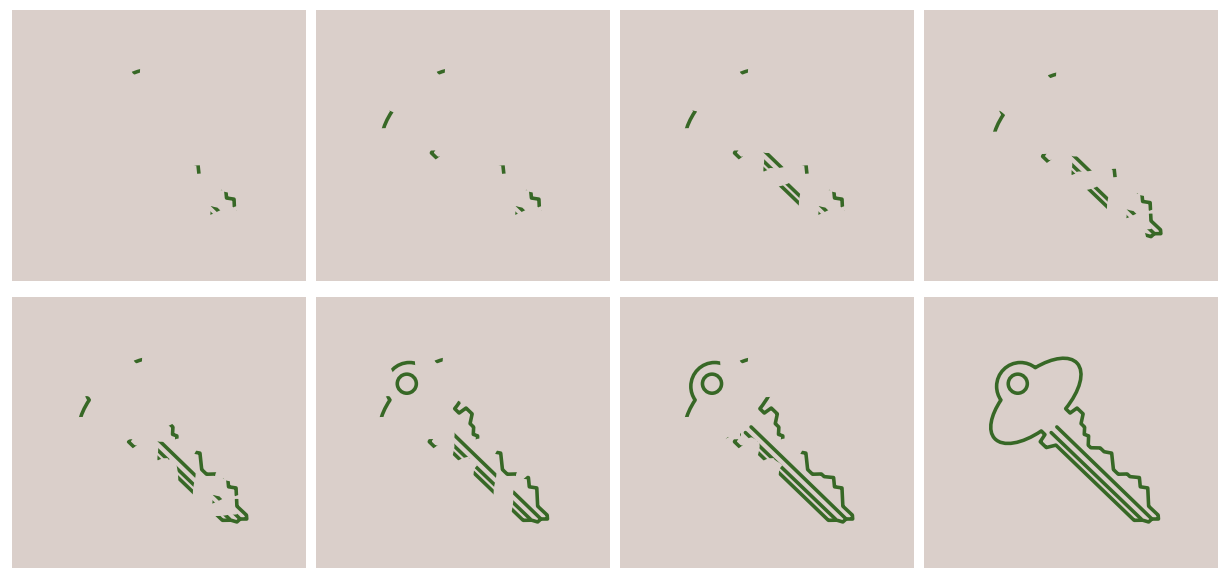

Fig. 1. Increasingly complete versions of a given object are shown until the object is identified. Source: Snodgrass et al. 1987; reproduced by permission from Life Science Associates.

the party exemplify the sensory-motor type of procedural memory.

\section{Episodic recall and recognition}

Laboratory tests of episodic memory generally involve a study phase for a set of to-be-remembered words (or pictures or sentences), followed by a test phase in which participants are asked to either recall or recognize the items that they recently studied. Recall tests can be either "free" (e.g., "Tell me everything you remember from the list") or cued (e.g., "Tell me all animals that you can remember from the list"). Age-related declines in episodic recall are well documented (fig. 2). Recognition tests typically require a "yes" versus "no" response as to whether an item was in the previously studied list. Older adults show little or no deficit in memory performance for recognition, in contrast to significant deficits for recall (fig. 3). This reduction has been attributed to environmental support, which reduces the effort needed for retrieval processes. The dissociation between recall and recognition is why we often fail to recall the name of a movie we saw or a person we met several days ago, whereas we can easily recognize the name as correct when it is presented to us either alone or as one of several options. The older we are, the more often we have failures of recall. However, when recognition is successful for a name that cannot be recalled, this shows that the name is at least partially available in memory but not readily accessible.

The differential susceptibility of recognition versus recall performance in normal aging is consistent

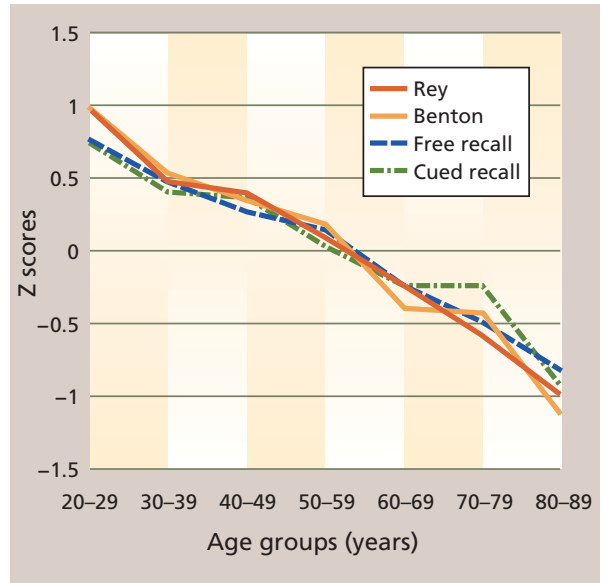

Fig. 2. Adult life span (cross-sectional) performance on four episodic memory tasks. "Benton" and "Rey" data are from clinical, pictorial, word-list learning tests named after the authors; "cued recall" and "free recall" data are from laboratory, word-list learning tasks created by the source author. Plotted $Z$ scores are referenced to the entire age range, with an overall mean of zero; younger adults are above and older adults are below zero. Source: Park et al. 2002; ๑ 2002 American Psychological Association.

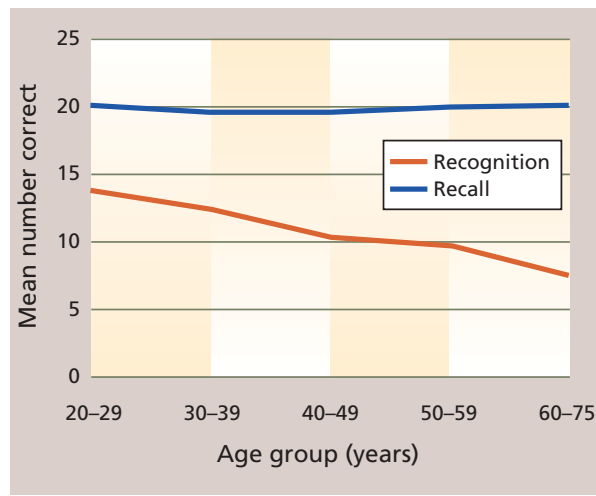

Fig. 3. Adult life span (cross-sectional) performance for free recall compared to recognition on a word-list learning, episodic memory task. Data source: Schonfield and Robertson 1966. 


\section{As we progress through middle and older age we acquire larger vocabularies and more extensive knowledge about the world around us.}

with dual-process models of episodic memory - recollection and familiarity (Light et al. 2000). Recollection is a relatively slow and deliberate process that involves memory access for the desired item/episode in tandem with information concerning the context in which it was experienced, such as when or where the episode occurred, the emotions experienced at the time, and so on. Familiarity, in contrast, is a relatively automatic process that provides a quick indication of the strength of the memory for the item/episode, without any link to the context in which it was experienced. For example, when you realize that you know the woman in front of you in a store's checkout line but cannot recall her name or where or when you met her, you are exhibiting successful familiarity-based memory, along with a frustrating lack of recollection-based memory. For both everyday and laboratory memory tasks, recollection plays a larger role in recall, whereas familiarity plays a larger role in recognition. Moreover, numerous studies indicate

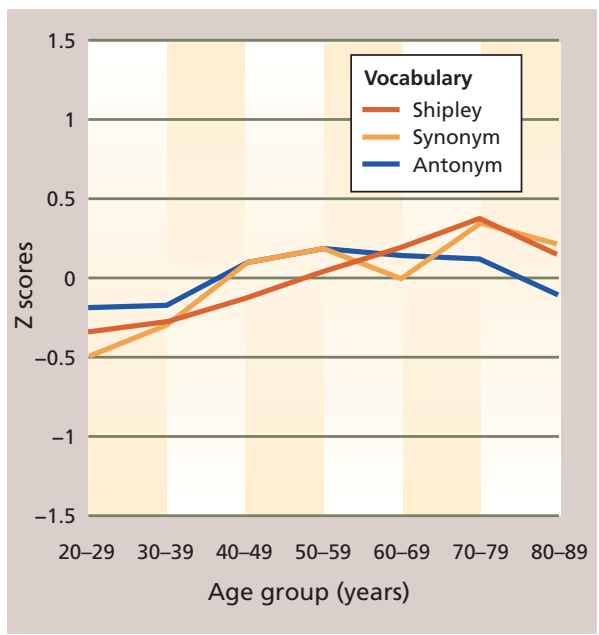

Fig. 4. Adult life span (cross-sectional) performance on three semantic memory tasks. "Shipley" data are from a standardized, clinical vocabulary test, whereas "synonym" and "antonym" data are from laboratorybased vocabulary tests. Plotted Z scores are referenced to the entire age range, with overall mean of zero. Performance improves until about age 70, followed by minimal decline. Source: Park et al. 2002; § 2002 American Psychological Association. that recollection is more negatively affected than familiarity, just as recall is more negatively affected than recognition, in aging. These two components of episodic memory may reflect neuroanatomically distinct memory processes, with recollection being more dependent on the brain's hippocampus, and familiarity being more dependent on the nearby entorhinal cortex.

\section{Semantic memory improves with age}

In contrast to the steady declines in episodic memory across all decades of adulthood, semantic memory is not only preserved, but also shows improvement until around the eighth decade of life, after which a gradual decline can be observed. Vocabulary and general knowledge tests are most commonly used to evaluate semantic memory (fig. 4). There is evidence that as we progress through middle and older age we acquire larger vocabularies and more extensive knowledge about the world around us, which is certainly good news regarding memory and aging. Increased world knowledge presumably can compensate to some extent for decreased facility in episodic memory. For example, linking to-be-remembered items and events in a meaningful way with information stored in semantic memory increases the likelihood of successful memory retrieval for these items; this type of semantic encoding strategy benefits older as well as younger adults (Froger et al. 2009).

Although our vocabulary knowledge is relatively protected from age-related loss, the ease with which we retrieve words is not. We are all familiar with the "tip-of-the-tongue" (TOT) phenomenon in our everyday lives. It begins when we are attempting to come up with a word, generally a noun and sometimes a proper name, that we want to use in conversation. We fail to retrieve the desired word or name, and yet we are sure that we know the word, and sometimes can think of the first letter, the last syllable or some aspect of

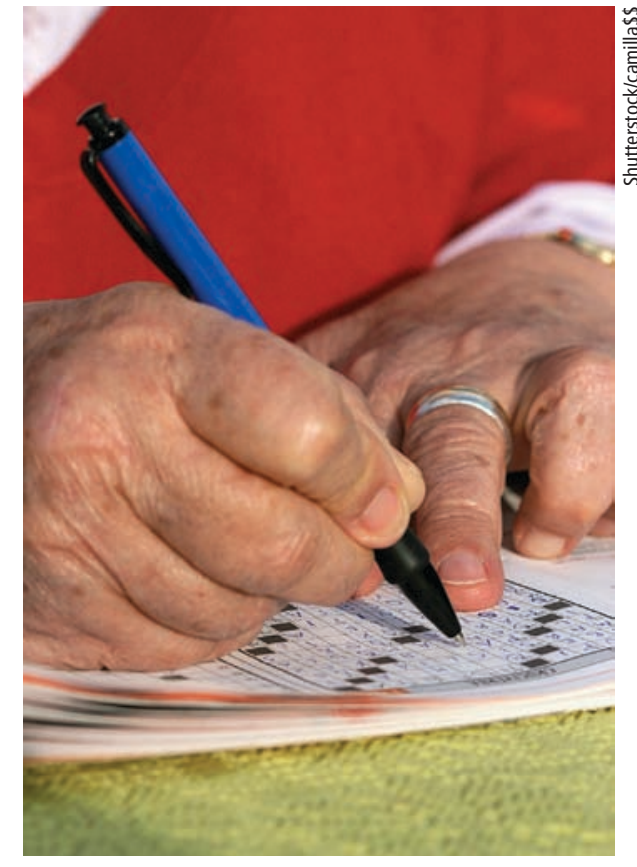

While episodic memory (the ability to remember things that happened) declines with age, semantic memory (one's store of general knowledge) continually increases until about the eighth decade of life.

the word's meaning. Sometimes, when we are not trying to retrieve the word, it simply "pops" into consciousness. TOTs have been studied with naturalistic diary studies as well as controlled laboratory methods (Burke et al. 1991; Heine et al. 1999). Findings indicate greater numbers of TOTs and longer resolution times for older compared to younger adulthood; however, without time pressure, older adults are as successful as younger adults in retrieving desired words. According to the widely favored incomplete activation (transmission deficit) hypothesis (Burke et al. 1991), TOTs occur when there is not enough activation from the semantic (meaning-based) knowledge about a desired word to lexical (word-based) and/or phonological (sound-based) knowledge, precluding the ability to retrieve the complete phonology for the desired word. This incomplete activation from semantics to phonology is more likely to happen for words and names encountered infrequently or not encountered recently, and for older as compared to younger adults. As frustrating as word-finding difficulties can be, the good news is that they are due to the slowing of access and retrieval, rather than the loss of knowledge. 
Alternate methods of assessing semantic memory do not require word retrieval and therefore are not affected by aging-related word-retrieval difficulties. These are referred to as implicit methods because the person being tested need not be consciously aware of the nature of the semantic knowledge being evaluated. A research method called "semantic priming" capitalizes on the fact that we are faster to process an item that follows an associated, semantically related item versus an unrelated item. For example, the word "truck" is processed more quickly when it follows "car" than when it follows "lion"; in this case the related words share features common to vehicles. The task required of the participants is most commonly a "word" versus "nonword" button press for each of a large number of letter strings (e.g., peach, salfay, car, truck). The timing of word presentation and recording of response times are computer controlled. The participant's attention is focused on the required response, rather than possible linkages between adjacent words, especially if the number of semantic linkages within the list is low. A reduction in response time, when associative and semantic linkages are present versus absent, comprises the semantic priming effect. With semantic priming tasks, the preservation of a wide variety of semantic knowledge about objects and their relationships has been demonstrated, not only for healthy elderly adults but also for patients with Alzheimer's disease (Ober 2002; Ober et al. 1991).

\section{Procedural memory preserved}

Procedural memory seems to be quite well preserved in normal aging. For sensory-motor tasks such as knitting or bicycle riding, although memory for sequential components is preserved these tasks may be performed more slowly and/or awkwardly due to motor performance issues associated with aging (e.g., arthritis, muscle weakness). For tasks that involve learning and memory for sequences or rules, but little or no motor skill, there is strong evidence for age-equivalent rates of learning and memory retention. Examples include card games such as bridge or solving Sudoko puzzles. Finally, for procedural-memory tasks that are more perceptual, such as picture or word identification (e.g., from an incomplete picture; fig. 1), older and younger adults show equivalent increases in speed and accuracy in identifying previously experienced pictures or words as compared to new ones.

\section{Brain and memory}

Multiple regions of the brain are involved in memory, with particular regions playing key roles in particular types of memory (figs. 5A and 5B). The episodic memory system depends critically on the medial temporal lobes (including the hippocampus and adjacent entorhinal and parahippocampal regions). Evidence for the hippocampus's critical role in episodic memory has come from neuroimaging studies of amnesia patients (e.g., post-stroke) showing damage to the hippocampus,
(A)

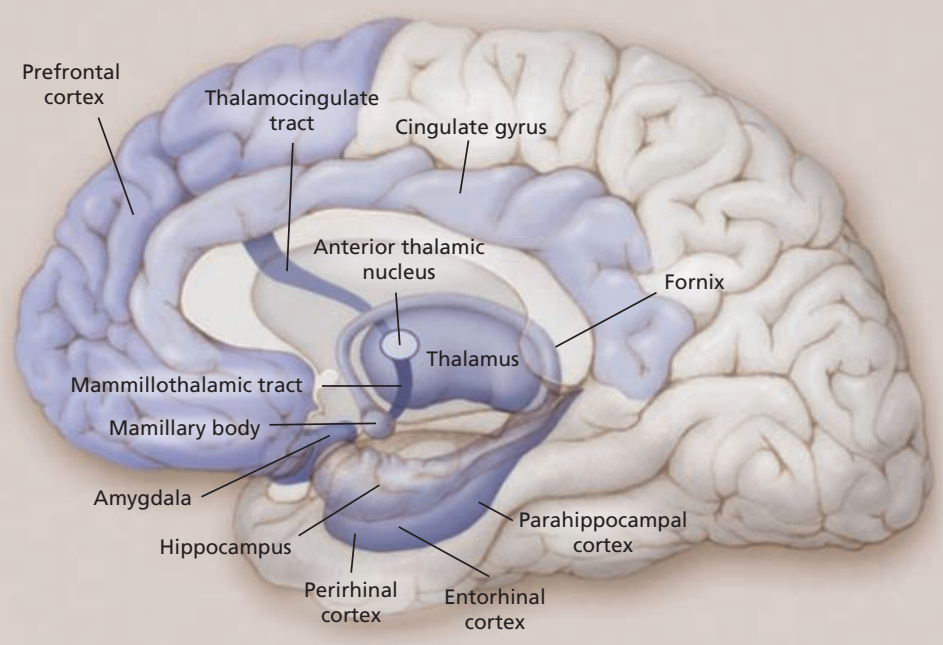

(B)

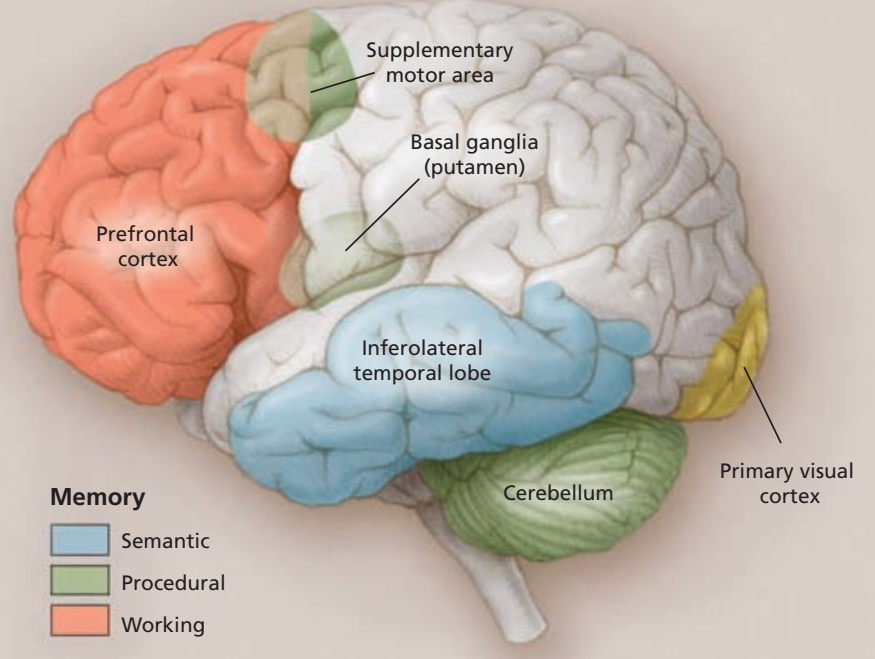

Fig. 5. (A) Areas of the brain significantly involved in episodic memory, shown from a midline view of the brain. The medial temporal lobes, including the hippocampus and parahippocampal cortex, are critical for normal episodic memory functioning, as are other brain regions highlighted in blue. (B) Areas of the brain significantly involved in semantic, procedural and working memory as shown from a lateral (external) view of the brain. Temporal lobe areas involved in semantic memory are different from those involved in episodic memory. Brain regions involved in procedural memory do not overlap with those involved in either episodic or semantic memory. The prefrontal cortex is involved in working memory and, in turn, encoding information into, and retrieving information from, long-term episodic and semantic memory. Source: Budson and Price 2005; ๑ 2005 Massachusetts Medical Society. All rights reserved. 
as well as dementia patients with prominent episodic memory deficits (Alzheimer's disease).

Even damage to a very small section of the hippocampus (such as can occur after $\mathrm{CO}_{2}$ poisoning or cardiac arrest) can result in significant episodic memory problems (Hopkins et al. 1995; Zola-Morgan et al. 1986). The hippocampus is part of the limbic system, and when a lesion occurs in particular limbic structures (e.g., mammillary body, fornix, anterior thalamic nucleus; fig. 5A) episodic memory dysfunction may occur. The prefrontal cortex (the large, anterior portion of the frontal lobes) is another important brain area for episodic memory; the hippocampal region needs to "work with" the frontal lobes (involving working memory processes). The hippocampal-frontal collaboration enables effective episodic encoding and retrieval, particularly for source (e.g., location, person, place) and time-related aspects of to-be-remembered events.

The semantic memory system is not dependent on the hippocampus or related structures, and world knowledge acquired prior to brain injury or disease is generally intact in amnesics. There is ample evidence that the visual features of objects are stored in the visual processing centers of the brain, auditory features are stored in the auditory processing centers of the brain, and so on, such that the complete knowledge representations of objects are distributed throughout the cortical regions involved in object perception. Additionally, specific regions of the left prefrontal cortex and bilateral anterior temporal cortex seem to be involved in retrieving, maintaining and selecting semantic information (Badre and Wagner 2002; Oliver and ThompsonSchill 2003).

Procedural memory, like semantic memory, is not dependent on the hippocampus or related structures; indeed, amnesic patients do not show impairments in the various types of procedural learning and memory tasks described earlier. The brain areas involved (e.g., motor cortex, visual cortex, prefrontal cortex and cerebellum; fig. 5B) are varied, and depend on the particular type of procedural learning and memory task.

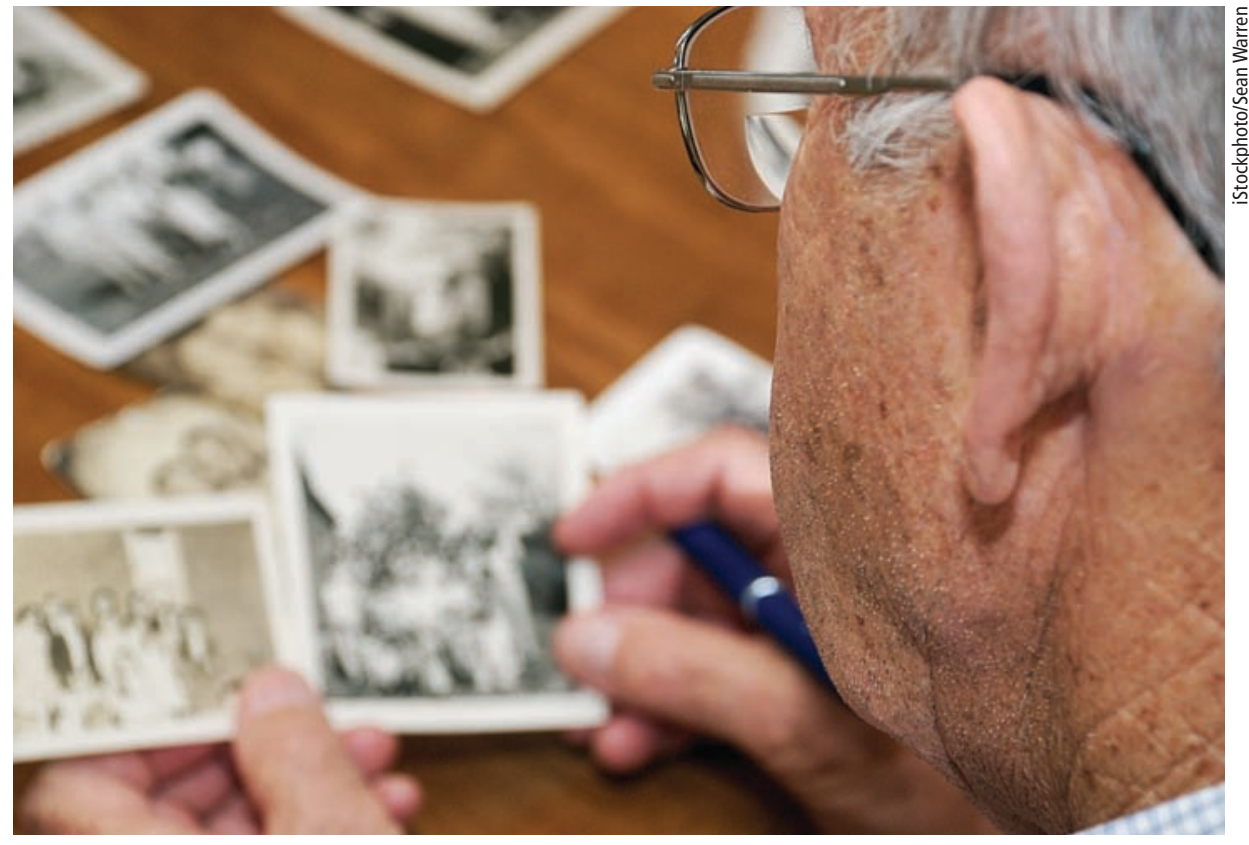

Certain parts of the brain show volume reductions with age, especially the prefrontal cortex and hippocampus. Both are important to the functioning of episodic memory, which plays a critical role in remembering past events.

\section{Structural brain changes}

Based on autopsy data and lowresolution (in vivo) CT scans, the gross structural brain changes associated with normal aging include: overall shrinkage of the cortex (brain atrophy due to tissue/cell loss), enlargement of the cerebral ventricles (structures in the center of the brain filled with cerebral spinal fluid), and increasing size of the cortical sulci (convolutions of the cerebral cortex that form "hills" [gyri] and "valleys" [sulci]). High-resolution magnetic resonance imaging (MRI) has enabled increasingly precise quantification of not only general brain changes, but also age-related volume changes for particular brain regions and structures.

Raz et al. (2005) summarized structural brain changes in normal aging. This cross-sectional study of adults ranging from about 20 to 80 years old also had a longitudinal component in that subjects were measured initially and after five years. For brain-volume changes both within individuals and across individuals of different ages, the hippocampus and subregions of the prefrontal cortex showed sizable reductions with age, whereas the entorhinal cortex and primary visual cortex showed minimal or no such reductions (fig. 5). The differential loss of volume for hippocampal versus entorhinal cortex is in keeping with evidence for greater dependence of recollection aspects of episodic memory (impaired in aging) on the hippocampus, versus greater dependence of familiarity aspects of episodic memory (relatively preserved in aging) on the entorhinal cortex; however, the entorhinal cortex does show volume loss among 70-plusyear-olds. When the integrity of white matter tracks (bundles of axons, which are parts of nerve cells that transmit electrical signals to other neurons) is specifically evaluated, either via MRI or diffusion tensor imaging (a highresolution imaging tool specifically targeted to white matter), the greatest deficiencies for older compared to younger adults are in the frontal lobes rather than the more posterior lobes (Head et al. 2004; Wen and Sachdev 2004).

To summarize, the structural brain changes associated with normal aging consist of declining brain tissue volume, with the prefrontal cortex and hippocampus particularly affected. The hippocampus plays an essential role in episodic memory functioning, and also must "work with" the prefrontal cortex in order for episodic encoding and retrieval to work effectively. Moreover, among the three types of long-term memory, only episodic memory is negatively affected in normal aging. 


\section{Brain function and plasticity}

Although there is ample evidence for decreases in tissue volume during the course of normal aging, three important facts greatly mitigate this bad news.

Atrophy. First, older adults are quite heterogeneous and some will have minimal brain-tissue loss over time. The degree of brain atrophy in normal aging may have been overestimated in previous research, because subjects with preclinical dementia were not excluded due to lack of long-term follow-up evaluation. Burgmans et al. (2009) began a longitudinal study with cognitively healthy adults (52 to 82 years old), and after three years an MRI brain scan accompanied cognitive testing. The subgroup of participants who had shown cognitive decline over the past three years exhibited a significant age-related decrease in brain tissue volume (for parahippocampal, cingulate gyrus and prefrontal cortex), but the subgroup who remained cognitively stable showed no age-related decreases. This promising finding will hopefully be replicated and extended.

Plasticity. Second, brain plasticity, which enables changes in brain structure and function due to experience and learning, occurs even in older adults. The brains of older as well as younger animals (rats and nonhuman primates) show increases in the number of neural synapses (by which neurons communicate with one another), neurotransmitter activity, number of glial cells (which support neuronal functions) and capillary vascularization (blood supply for neurons and glial cells) as a function of "enriched environments" or specific learning experiences (Kramer et al. 2004; Markham and Greenough 2004). Using methodologies appropriate to human subjects (functional MRI), researchers have demonstrated longlasting changes in regional brain activation patterns as a result of "targeted" behavioral training.

The most exciting research on brain plasticity, however, provides evidence for neurogenesis (the "birth" of new neurons), not only in adult nonhuman animals, but in older, as well as younger, adult human brains, as a function of new learning (Kempermann 2006). The brain areas where neurogenesis has been reliably demonstrated are the hippocampus, olfactory bulb (where primary odor processing occurs) and caudate nucleus (near the center of the brain and involved in feedback-related aspects of learning and memory). Hippocampal neurogenesis is especially good news, given the critical role of the hippocampus in laying down new episodic memories, and given that the hippocampus is one of the most vulnerable brain areas in terms of cell loss associated with normal aging (and to a much greater extent, with Alzheimer's disease).

Bilaterality. Third, brain function and the cognitive functions dependent upon it may not be in "lock-step" with decreases in brain tissue volume, due to age-related changes in the patterns of brain activation that are associated with particular types of cognitive tasks. One such age-related change is the increased bilaterality (activation in both hemispheres) of prefrontal activations in older as compared to younger adults (Park and Reuter-Lorenz 2009). In younger adults, verbal memory tasks result in focal activations in the left prefrontal cortex, whereas visual/spatial memory tasks result in right prefrontal activations. This strong laterality is not seen in older adults; instead, activation in the "appropriate" side of the brain is reduced, while activation in the "inappropriate" side is increased. Increased bilaterality has been widely observed and it seems to be compensatory, enabling improved performance: older adults who engage both the left and right prefrontal cortex on memory tasks have higher performance than those who don't show bilateral activation (the improved performance is closer, but not equal to, that seen for younger adults).

\section{Maintaining brain function}

The question of how to maintain brain function into older age has captured the attention of many thousands of biological and behavioral scientists nationally and internationally, who are obtaining large amounts of research funding from government sources, industry and private foundations. In fact, UC scientists authored 1,885 articles on human brain aging and/or cognitive aging from 2000 to 2009, per the Web of Science (an online academic citation index by Thomson Reuters). As the older adult population continues growing at the state, national and global levels, there is increasing interest in interventions to minimize brain atrophy, maximize brain functioning, and maintain adequate memory and other cognitive functions throughout later adulthood. The extent that memory and other cognitive functions can be maintained, or at least minimally impaired, will have positive effects on day-to-day functioning and life satisfaction for older adults, as well as reduce caregiving costs and extend the years of independent living (i.e., fewer years in a nursing home).

Physical activity. There is increasing support for the hypothesis that exercise as well as general physical fitness can improve cognitive (including memory) and brain function across the adult life span. Initial evidence was based mainly on observational studies. One particularly impressive correlational study (Erickson et al. 2009) showed that higher cardiovascular fitness significantly predicted greater MRI-measured hippocampal volume, as well as performance on an episodic memory task, in a large sample of 59- to 81-year-olds, even after controlling for age, sex and years of education.

In recent years, a number of exercise intervention studies have enabled the evaluation of causal links between exercise and cognition. These studies

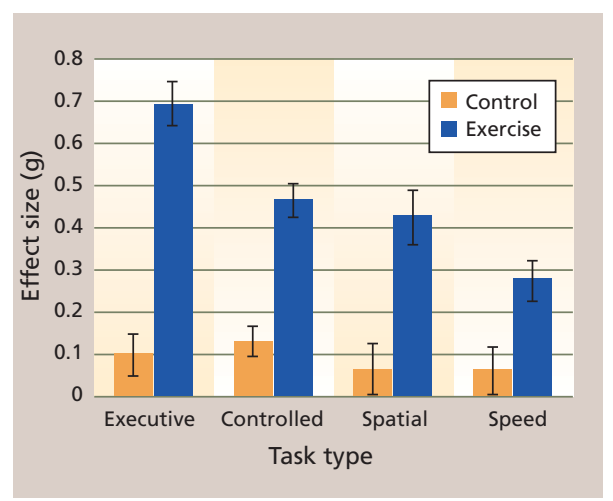

Fig. 6. Effect sizes of mean differences in cognitive-task performance between older adults in an aerobic fitness program and those who were not (control). Executive = planning and scheduling of mental processes; controlled = inhibiting one response in order to "deliver" the desired response; spatial = remembering visuospatial information; and speed = low-level reaction time. Source: Colcombe and Kramer 2003; ๑ 2003 Assoc. Psychological Science. Reproduced by permission of SAGE Pubs. 


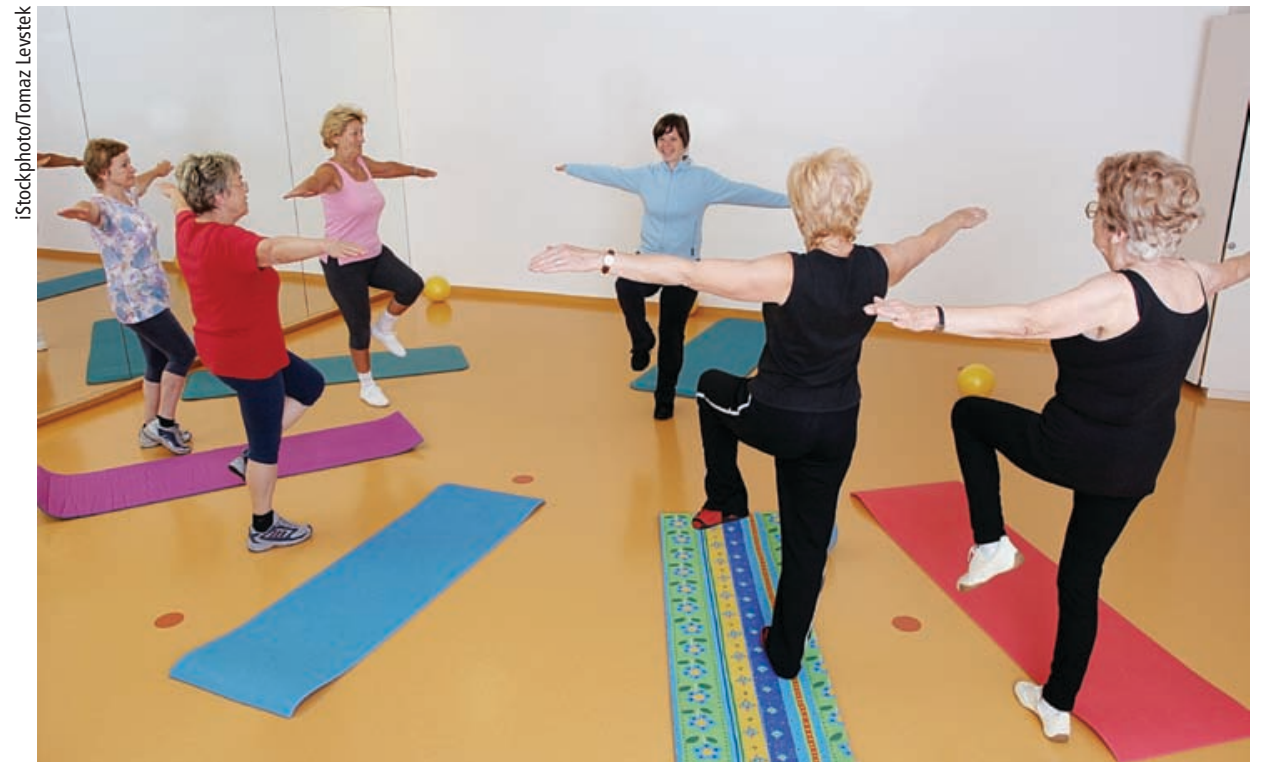

A recent study showed that higher cardiovascular fitness among 59- to 81-year-olds was correlated with greater volume in the brain's hippocampus and better performance in an episodic memory task.

involve the random assignment of participants to an exercise or control (no exercise) group. Both before the start and at the end of treatment, various aspects of cognitive functioning are assessed. Most, but not all, individual studies have shown that exercise benefits cognition. Fortunately, several meta-analyses have been carried out, which involve the calculation of exercise (vs. control) effects for many individual studies (meeting predetermined criteria) and then obtaining overall effect sizes for cognitive measures shared across stud-

\section{The "use it or lose it"}

\section{hypothesis is that increasing amounts of mental activity enable higher levels of cognitive functioning in middle and late adulthood.}

ies. The upshot is that exercise positively affects cognitive functioning in adults 55 and older, even those with early dementia (Kramer and Erickson 2007).

Overall effect sizes in these metaanalyses tend to be around 0.5 , which is moderately robust, as in Colcombe and Kramer (2003) (fig. 6). For each of four cognitive tasks, the effect was statistically significant for the exercise but not control participants; episodic memory tasks were part of the "visuospatial" task domain. Additional findings included: a larger effect of exercise training when the program duration was greater than 6 months, as opposed to 1 to 3 or 4 to 6 months; a much larger effect for training sessions that were more than 30 minutes, as opposed to 15 to 30 minutes long; a somewhat larger effect for training that combined cardiovascular and strength training, as opposed to cardiovascular training alone; and finally, much larger training effects for the older age groups (66 to 70, or 71 to 80 years) as compared to the younger age group (55 to 65 years).

"Use it or lose it." The "use it or lose it" hypothesis is that increasing amounts of mental activity enable higher levels of cognitive functioning in middle and late adulthood; in other words, mental activity can counteract the effects of normal aging. Early research on mental activity was dominated by correlational, observationbased studies. These studies involve the collection of self-report data from large numbers of older adults regarding their engagement in activities presumed to be mentally stimulating (e.g., reading books, playing bridge, solving crossword puzzles), with follow-up over varying periods of time to assess cognitive status. The findings strongly support a positive correlation of mental activity at baseline with overall cognitive function, including a reduced risk of Alzheimer's disease, at follow-up evaluations spanning five or more years. For example, Wilson et al. (2007) found that a low mental activity group was 2.5 times more likely to develop Alzheimer's disease than a high mental activity group over 5 years. Another example is a report by Valenzuela et al. (2008) that older adults' scores on a lifetime mental activity questionnaire were significantly predictive of MRI-assessed hippocampal atrophy over the following 3 years; that is, there is less atrophy with higher mental activity scores. Although encouraging, the correlational nature of these findings does not permit us to conclude that mental activity causes improved cognitive or brain outcome. It could be that more mentally active adults have healthier brains and/or have been more mentally active in the years prior to the start of the study than the less mentally active adults.

Increasing numbers of studies directly manipulate cognitive activity via training sessions designed to improve episodic memory and other cognitive functions (Hertzog et al. 2009). There is ample evidence for the ability of older adults to show improvement on episodic memory tasks (as well as many other cognitive tasks) with practice or training, especially when training in the use of strategies is involved. Evidence is limited and at times conflicting regarding how long-lasting the effects of training are, the degree to which it can be generalized to nontrained but similar tasks, and whether the training is actually resulting in a reduction of age-related deficits on the trained task (as opposed to benefitting older and younger adults equally, thus maintaining the same degree of agerelated deficits).

Among the "gold standard" type of cognitive intervention, the most ambitious is the ACTIVE (Advanced Cognitive Training for Independent and Vital Elderly) project, a multisite study in which 2,802 adults over 65 years of age were randomly assigned to a notraining (control) group or one of three cognitive training groups, with training taking place over 10 twice-per-week sessions. Each training group focused on one of the following: memory (episodic 
list-learning), reasoning or speed of processing. All participants were tested in all three cognitive domains at the end of the training period, and yearly thereafter. Each of the three types of cognitive training yielded improved performance in the cognitive domain being trained (e.g., memory training improved memory performance); however, there was no transfer of these training effects to cognitive domains that were not trained (Ball et al. 2002). The largest training effects occurred for speed of processing, and the smallest were for memory. Five-year follow up data showed that the initial training benefits were still present, albeit much reduced (Willis et al. 2006).

Salthouse's (2006) review of the mental activity literature concluded that although cognitive interventions can improve performance, there is a dearth of evidence for the transfer of training to untrained tasks (even within the same cognitive domain, such as episodic memory), or for long-lasting benefits. Based on a meta-analysis of randomized control trials of cognitive intervention, many of which included memory training, Papp et al. (2009) also concluded that there was no evidence for the generalizability or long-term benefits of training, with the important caveat that few studies have incorporated long-term follow-up. However, the vast majority of cognitive intervention studies have entailed fewer than 10 training sessions; it is possible that with a larger number of training sessions, significant, long-term benefits could be obtained.

On a more positive note, an intriguing experimental field study on cognitive training by Stine-Morrow et al. (2008) provided evidence that older adults who are immersed in a 6-month program of group-based, creative problem-solving ("Senior Odyssey," modeled after the international "Odyssey of the Mind" program for children and young adults) show positive changes in fluid cognitive abilities (i.e., the processing and manipulation of information) that were not explicitly trained via the Odyssey program. More specifically, the Odyssey participants showed significant increases in performance in comparison to controls for post-test ( 7 to 8 months after the start of the Odyssey program) compared to pretest (prior to the start of the program) assessments of the speed of information processing, inductive reasoning and divergent thinking (e.g., generating word associations or coming up with alternate/unusual uses of objects). The authors acknowledge that these improved cognitive abilities may not be maintained over a longer period of time in the absence of further engagement in the Odyssey or some similar program. Nonetheless, these findings demonstrate the transfer of cognitive training/ engagement to thinking and reasoning skills that were not specifically trained.
Further work is needed to determine whether memory skills, especially episodic memory (not assessed in the Stine-Morrow [2008] et al. study) could also be improved by the type of groupbased, intensive, cognitive training exemplified by the Odyssey program.

\section{Understanding memory, brain and aging}

During the past 10 to 15 years, major advances have been made in our understanding of the nature of memory changes that occur in normal aging, as well as the brain underpinnings for these changes. There have also been major advances in our understanding

\section{Tips for maintaining and, perhaps, improving brain and memory functions in middle-to-older adulthood}

- Get regular medical checkups and make it a lifelong goal to maintain healthy blood pressure, weight and cholesterol levels.

- Remind yourself, regularly, that "What is good for my heart is also good for my brain."

- Engage in challenging and interesting cognitive activities (e.g., start learning, or re-learning, a second language).

- Exercise regularly (e.g., 30 minutes of brisk walking daily).

- Participate in social activities, via community organizations, special-interest clubs, etc.

- Take up dancing (this activity has physical, mental and social components) or some other "multicomponent" activity.

- Eat a well-balanced diet with plenty of fruits and vegetables (supplying vitamins and minerals that are important for brain function).

- Eat fish that is high in omega-3 fatty acids (e.g., salmon and trout) several times per week, or take a daily supplement for omega-3 fatty acids.

- Keep stress levels as low as possible (meditation, yoga and exercise can all help with stress reduction).

- Get 7 to 8 hours of sleep per night.

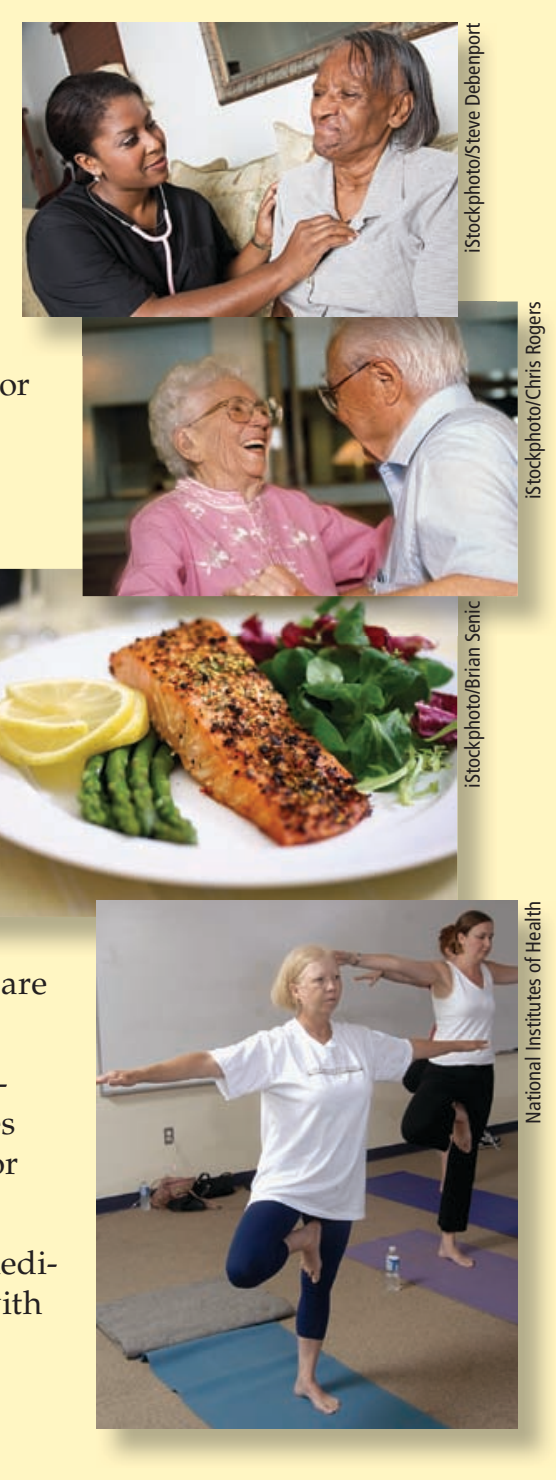


of the variability in neurocognitive aging, including, on the positive end of the continuum, documentation of older adults who show no brain or cognitive changes over several years (Burgmans et al. 2009). Evidence for brain plasticity in late adulthood continues to grow; this includes findings of neurogenesis as well as age-related changes in patterns of brain activation that are associated with improved cognitive performance.

Generally, age-related increases in brain activations (e.g., frontal lobes) are interpreted as compensating for shrinkage in numerous brain structures (e.g., hippocampus) and declines in some

\section{References}

Badre D, Wagner AD. 2002. Semantic retrieval, mnemonic control and prefrontal cortex. Behav Cogn Neurosci Rev 1(3):206-18.

Ball K, Berch DB, Helmers KF, et al. 2002. Effects of cognitive training interventions with older adults: $A$ randomized controlled trial. JAMA 288(18):2271-81.

Brookmeyer R, Johnson E, Ziegler-Graham K, Arrighi HM. 2007. Forecasting the global burden of Alzheimer's disease. Alzheimer Dementia 3:186-91.

Budson AE, Price BH. 2005. Current concepts - memory dysfunction. New Engl J Med 352(7):692-9.

Burgmans S, van Boxtel MPJ, Vuurman EFPM, et al. 2009. The prevalence of cortical gray matter atrophy may be overestimated in the healthy aging brain. Neuropsychology 23(5):541-50.

Burke DM, MacKay DG, Worthley JS, Wade E. 1991. On the tip of the tongue - what causes word-finding failures in young and older adults? J Mem Lang 30(5):542-79

Colcombe S, Kramer AF. 2003. Fitness effects on the cognitive function of older adults: A meta-analytic study. Psychol Sci 14(2):125-30

Erickson KI, Prakash RS, Voss MW, et al. 2009. Aerobic fitness is associated with hippocampal volume in elderly humans. Hippocampus 19(10):1030-9.

Froger C, Taconnat L, Landre L, et al. 2009. Effects of level of processing at encoding and types of retrieval task in mild cognitive impairment and normal aging. J Clin Exp Neuropsychol 31(3):312-21

Head D, Buckner RL, Shimony JS, et al. 2004. Differential vulnerability of anterior white matter in nondemented aging with minimal acceleration in dementia of the Alzheimer type: Evidence from diffusion tensor imaging. Cereb Cortex 14(4):410-23.

Heine MK, Ober BA, Shenaut GK. 1999. Naturally occurring and experimentally induced tip-of-the-tongue experiences in three adult age groups. Psychol Aging 14(3):445-57.

Hertzog C, Kramer AF, Wilson RS, Lindenberger U 2009. Enrichment effects on adult cognitive development. Can the functional capacity of older adults be preserved and enhanced? Psychol Sci Public Interest 9:1-65. aspects of cognition (e.g., episodic memory). Indeed, the Scaffolding Theory of Aging and Cognition is based on compensatory scaffolding, that is, the recruitment of additional neural networks to support declining networks whose functions have become inefficient and/ or noisy (Park and Reuter-Lorenz 2009). The challenge going forward is to further advance our understanding of the biological as well as psychological aspects of memory functioning in normal aging, such that specific lifestyle (such as exercise regimen and dietary supplements) and pharmacological treatments can be recommended to middle-aged and older adults, with a high likelihood

Hopkins RO, Kesner RP, Goldstein M. 1995. Item and order recognition memory in subjects with hypoxic brain injury. Brain Cognition 27(2):180-201.

Kempermann G. 2006. Adult Neurogenesis: Stem Cells and Neuronal Development in the Adult Brain. New York, NY: Oxford Univ Pr. 448 p.

Kramer AF, Bherer L, Colcombe SJ, et al. 2004. Environmental influences on cognitive and brain plasticity during aging. J Gerontol: A-Biol Sci 59(9):940-57.

Kramer AF, Erickson KI. 2007. Capitalizing on cortica plasticity: Influence of physical activity on cognition and brain function. Trend Cogn Sci 11(8):342-8.

Light LL, Prull MW, La Voie DJ, Healy MR. 2000. Dualprocess theories of memory in old age. In: Perfect TJ, Maylor EA (eds.). Models of Cognitive Aging: Debates in Psychology. New York, NY: Oxford Univ. Pr.

Markham JA, Greenough WT. 2004. Experience-driven brain plasticity: Beyond the synapse. Neuron Glia Biol 1(4):351-63

Ober BA. 2002. RT and non-RT methodology for semantic priming research with Alzheimer's disease patients: A critical review. J Clin Exp Neuropsyc 24(7):883-911

Ober BA, Shenaut GK, Jagust WJ, Stillman RC. 1991 Automatic semantic priming with various category relations in Alzheimer's disease and normal aging. Psychol Aging 6(4):647-60

Oliver RT, Thompson-Schill SL. 2003. Dorsal stream activation during retrieval of object size and shape. Cogn Affect Behav Neurosci 3(4):309-22.

Papp KV, Walsh SJ, Snyder PJ. 2009. Immediate and delayed effects of cognitive interventions in healthy elderly: A review of current literature and future directions. Alzheimer Dementia 5:50-60.

Park DC, Lautenschlager G, Hedden T, et al. 2002. Models of visuospatial and verbal memory across the adult life span. Psychol Aging 17:299-320.

Park DC, Reuter-Lorenz P. 2009. The adaptive brain: Aging and neurocognitive scaffolding. Ann Rev Psychol 60:173-96.

Raz N, Lindenberger U, Rodrigue KM, et al. 2005. Regional brain changes in aging healthy adults: General trends, individual differences and modifiers. Cereb Cortex 15(11):1676-89. of these treatments leading to satisfactory memory performance and relatively independent living well into the ninth and tenth decades of life. Successful treatments would presumably be those that result in increased brain scaffolding, made possible by the multiple mechanisms of neural plasticity that continue to function in older adulthood.

B.A. Ober is Professor, Department of Human and Community Development, UC Davis. The Guest Associate Editor for this article was Lisa M. Soederberg Miller, Associate Professor, Department of Human and Community Development, UC Davis.

Salthouse TA. 2006. Mental exercise and mental aging: Evaluating the validity of the "use it or lose it" hypothesis. Perspect Psychol Sci 1(1):68-87.

Schacter DL, Tulving E. 1994. What are the memory systems of 1994? In: Schacter DL, Tulving E (eds.). Memory Systems 1994. Cambridge, MA: MIT Pr. p $1-38$

Schacter DL, Wagner AD, Buckner RL. 2000. Memory systems of 1999. In: Tulving E, Craik IM (eds.). The Oxford Handbook of Memory. Oxford Univ Pr. p 627-43.

Schonfield D, Robertson B. 1966. Memory storage and aging. Can J Psychol 20:228-36.

Snodgrass JG, Smith B, Feenan K, Corwin J. 1987 Fragmenting pictures on the Apple Macintosh computer for experimental and clinical applications. Beh Res Method Instrument Computer 19:270-4.

Squire LR, Knowlton BJ. 2000. The medial temporal lobe, the hippocampus and the memory systems of the brain. New Cognitive Neurosci:765-79.

Stine-Morrow EAL, Parisi JM, Morrow DG, Park DC 2008. The effects of an engaged lifestyle on cognitive vitality: A field experiment. Psychol Aging 23(4):77886.

US Census Bureau. 2010. State and County Quick Facts. http://quickfacts.census.gov/qfd/states/06000 html.

Valenzuela MJ, Sachdev P, Wen W, et al. 2008. Life span mental activity predicts diminished rate of hippocampal atrophy. PLoS One 3(7):e2598.

Wen W, Sachdev P. 2004. The topography of white matter hyperintensities on brain MRI in healthy 60-to64-year-old individuals. Neuroimage 22(1):144-54.

Willis SL, Tennstedt SL, Marsiske M, et al. 2006 Long-term effects of cognitive training on everyday functional outcomes in older adults. JAMA 296(23):2805-14.

Wilson RS, Scherr PA, Schneider JA, et al. 2007. Relation of cognitive activity to risk of developing Alzheimer's disease. Neurology 69:1911-20.

Zola-Morgan S, Squire LR, Amaral DG. 1986. Human amnesia and the medial temporal region - enduring memory impairment following a bilateral lesion limited to field CA1 of the hippocampus. J NeurosC 6(10):2950-67. 